\title{
Identification of suitable reference genes for BDV-infected primary rat hippocampal neurons
}

\author{
QIANG MAO ${ }^{1-3^{*}}$, LUJUN ZHANG $^{1-3^{*}}$, YUJIE GUO ${ }^{1,3^{*}}$, LU SUN $^{2,3^{*}}$, SIWEN LIU $^{2,3}$, PENG HE $^{2,3}$, \\ RONGZHONG HUANG ${ }^{4}$, LIN SUN ${ }^{3,4}$, SHIGANG CHEN ${ }^{2,3}$, HONG ZHANG $^{2,3}$ and PENG XIE ${ }^{1-3,5}$ \\ ${ }^{1}$ Department of Neurology, Yongchuan Hospital, Chongqing Medical University, Chongqing 402460; \\ ${ }^{2}$ Institute of Neuroscience and The Collaborative Innovation Center for Brain Science; \\ ${ }^{3}$ Chongqing Key Laboratory of Neurobiology; ${ }^{4}$ Department of Rehabilitation, The Second Affiliated Hospital; \\ ${ }^{5}$ Department of Neurology, The First Affiliated Hospital, Chongqing Medical University, \\ Chongqing 400016, P.R. China
}

Received October 1, 2015; Accepted September 26, 2016

DOI: $10.3892 / \mathrm{mmr} .2016 .5959$

\begin{abstract}
Borna disease virus (BDV) is a neurotropic RNA virus that infects the limbic system of mammals and results in behavioral disorders. The hippocampus is a core region in the limbic system, which contributes to memory and learning and is important in the regulation of emotion. However, no validated microRNA housekeeping genes have yet been identified in BDV-infected rat primary hippocampal neurons. Proper normalization is key in accurate miRNA expression analysis. The present study used reverse transcription-quantitative polymerase chain reaction (RT-qPCR) to evaluate the expression stability of 10 commonly used reference genes $[\mathrm{miR}-92 a, 5 \mathrm{~S}, \mathrm{U6}, \mathrm{miR}-103, \mathrm{miR}-101 a$, miR-let-7a, miR-16, E2 small nucleolar RNA (snoRNA), U87 and $m i R-191]$ in BDV-infected rat hippocampal neurons and non-infected controls across 12 days post-infection. The data was analyzed by four statistical algorithms: geNorm, NormFinder, BestKeeper, and the comparative $\Delta-C t$ method. Subsequently, the most suitable reference genes (miR-101a and U87) and the least suitable (snoRNA) were determined by the RankAggreg package. $m i R-155$ was selected as a standard by which to evaluate the most and least suitable reference genes. When normalized to the most stable reference gene there were significant differences between the two groups. However, when the data were normalized to the less stably expressed gene, the results were not significant.
\end{abstract}

Correspondence to: Professor Peng Xie, Department of Neurology, The First Affiliated Hospital, Chongqing Medical University, 1 Yixue Road, Chongqing 400016, P.R. China

E-mail: xiepeng@cqmu.edu.cn

*Contributed equally

Key words: BDV, limbic system, reference gene, miRNAs, qRT-PCR, hippocampal neurons
miR-101a was recommended as a suitable reference gene for BDV-infected rat primary hippocampal neurons.

\section{Introduction}

Borna disease virus (BDV) is an enveloped, non-segmented, negative-sense, single-stranded RNA virus, which belongs to the Bornaviridae family (1). BDV is a neurotropic that persists in the central nervous system (2), and affects infected individuals for their entire life span resulting in chronic, persistent infections of neurons and glial cells. It has been reported that BDV infects a range of animal species worldwide (3), including China (4,5). BDV infects neurons in the limbic system, and primarily in the hippocampus and the cortex, which has wide spread connections to diverse cortical areas (6). However, BDV infection is restricted to areas of the rat brain that are responsible for intensive neurodegeneration in newborn Sprague-Dawley rats, indicating that the function of immature neural cells is impaired by BDV (7).

Although the exact mechanism(s) of BDV pathogenesis have not been determined (8), there is interest in the interaction of host mRNA and microRNAs (miRNAs). miRNAs are short ( $22 \mathrm{nt})$ endogenous RNAs that are key in regulating expression of a diverse genes involved in different cellular processes. Aberrant miRNA expression has been associated with a number of human diseases and accurate quantification of miRNA expression is important for their use as biomarkers and to determine their functions in health. When attempting to analyze the consequences of BDV infection in a model, a well-established and understood technique was required, thus, the accurate and specific reverse transcription-quantitative polymerase chain reaction (RT-qPCR) is used to evaluate miRNA levels in biological samples (9).

There has been no formal evaluation of optimal primary rat hippocampal neurons miRNA reference genes for BDV research. Thus, the current study systematically identified the most stable housekeeping genes in cultured primary rat hippocampal neurons infected with BDV Hu-H1 strain. A total of 10 commonly used reference genes [5S, U6, U87, E2 small nucleolar RNA (snoRNA), miR-16, miR-103, miR-191, 
miR-let-7a, miR-92a and miR-101a] were selected as candidate reference genes, and RT-qPCR was subsequently used to validate the infected and non-infected hippocampal neurons. Four statistical algorithms and consensus ranking were applied to identify the most stable reference genes. These results may provide information regarding appropriate reference genes for normalization of RT-qPCR data in BDV-infected rat hippocampal neurons.

\section{Materials and methods}

Primary culture of hippocampal neurons and viral infection. Hippocampal neurons were isolated from the brains of 24 male Sprague-Dawley rats (postnatal day 1; weight, 8-10 g; Chongqing Medical University Animal Experimental Center, Chongqing, China). Prior to neuronal isolation the rats were anesthetized in ice for 1-5 min, after which they were incubated in $70 \%$ ethanol for $30 \mathrm{sec}$ until hyothermic; subsequently, the rats were decaptitated and the heads were placed in cold Hank's Balanced Salt Solution (HBSS; Invitrogen; Thermo Fisher Scientific, Inc., Waltham, MA, USA). The neurons were maintained in Dulbecco's modified Eagle's medium/Nutrient Mixture F-12 (Gibco; Thermo Fisher Scientific, Inc., Waltham, MA, USA) supplemented with $15 \%$ fetal calf serum, $10 \%$ horse serum, $1 \%$ glutamine, $0.5 \% 10 \mathrm{U} / \mathrm{ml}$ penicillin/streptomycin (all obtained from Gibco; Thermo Fisher Scientific, Inc.), as previously described (10). Cells were seeded at a density of $5 \times 10^{5}$ cells/well on poly-L-lysine-coated six-well plates (Sigma-Aldrich; Merck Millipore, Darmstadt, Germany). After $4 \mathrm{~h}$ at $37^{\circ} \mathrm{C}$, half of the cultured neurons were infected with BDV Hu-H1 strain [in an oligodendroglia cell line; multiplicity of infection $(\mathrm{MOI})=0.2]$, which was presented by Professor Hanns Ludwig (Free University of Berlin, Berlin, Germany), by adding cell-released virus (CRV) to the culture medium. CRV stocks were prepared as previously described (11). The BDV Hu-H1 strain is one of the first three human strains derived from mentally affected patients (12). These strains have been partially characterized by sequencing (13). The remaining neurons were maintained as a control sample. After $2 \mathrm{~h}$ of BDV infection, excess virus was removed by washing with HBSS ( $1 \mathrm{ml} /$ well; $\mathrm{Ca}^{2+}$ and $\mathrm{Mg}^{2+}$ free). Subsequently, the neurons were incubated in neurobasal medium with $2 \%$ B-27 (Gibco; Thermo Fisher Scientific, Inc.) for 12 days at $37^{\circ} \mathrm{C}$. The present study was approved by the ethics committee of Chongqing Medical University (Chongqing, China).

Immunofluorescence. To identify the purity of neurons and the effect of BDV infection for each experiment, a standard immunofluorescence protocol was used, as previously described $(14,15)$ and observed with a fluorescence microscope (Nikon Corporation, Tokyo, Japan). Briefly, the BDV-infected and non-infected neurons were incubated in six-well plates for $20 \mathrm{~min}$ at room temperature with $4 \%$ paraformaldehyde, followed by permeabilization for $10 \mathrm{~min}$ in $0.25 \%$ Triton $\mathrm{X}-100$. Subsequently, the two neuron groups were rinsed with HBSS ( $1 \mathrm{ml} /$ well) three times for $5 \mathrm{~min}$ and blocked with $5 \%$ bovine serum albumin (Sigma-Aldrich) for $30 \mathrm{~min}$, followed by incubation at $4^{\circ} \mathrm{C}$ overnight with primary antibodies (16). Following three washes with HBSS, the cells were incubated with the neuron-specific marker microtubule associated protein 2 (1:5,000; cat. no. ab5392; Abcam, Cambridge, MA, USA) and a BDV-specific anti-P40 monoclonal antibody (1:400; cat. no. AJ1572a; Abgent, Inc., Santa Diego, CA, USA) (15). Cells were then incubated with Alexa Fluor 594-conjugated goat anti-chicken secondary antibody (1:500; cat. no. A-11042; Invitrogen; Thermo Fisher Scientific, Inc.) for $2 \mathrm{~h}$ at room temperature. Following extensive phosphate-buffered saline washing, cells were incubated with DAPI (Beyotime Institute of Biotechnology, Haimen, China) for $1 \mathrm{~min}$ at room temperature and observed using an inverted fluorescence microscope.

RT-qPCR expression studies of candidate reference genes. A total of 10 commonly used candidate reference genes for miRNAs were selected from the reference literature (17-20). Their primer sequences, amplicon size, and amplification efficiencies are presented in Table I. To identify candidate reference miRNAs from each hippocampal neurons group, the following criteria were used: i) miRNAs had to be detected as 'present' (foreground-background, >100) in all 32 samples; ii) the fold-change of the candidate reference gene expression indicated a significant difference between the two groups $(\mathrm{P}<0.05)$; and iii) candidate miRNAs must be unambiguously annotated in the miRBase (http://www.mirbase.org/).

RNA isolation and reverse transcription. Total RNA was extracted from the hippocampal neurons using RNAiso Plus (Takara Bio, Inc., Otsu, Japan) according to the manufacturer's protocols. The samples were dissolved in $20 \mu \mathrm{l}$ DNase/RNase-free $\mathrm{H}_{2} \mathrm{O}$ and stored at $-80^{\circ} \mathrm{C}$ until use. Isolated RNA was reverse transcribed into cDNA with GoScript ${ }^{\mathrm{TM}}$ Reverse Transcription system (Promega Corporation, Madison, WI, USA) according to the manufacturer's protocols. Briefly, the first reaction mixture consisted of $4 \mu \mathrm{l}$ RNA, $5 \mu \mathrm{l}$ total primers, and $1.6 \mu \mathrm{l}$ DNase/RNase-free $\mathrm{H}_{2} \mathrm{O}$ performed in a Gene Amp PCR System 9700 (Applied Biosystems; Thermo Fisher Scientific, Inc.) at $42^{\circ} \mathrm{C}$ for $15 \mathrm{~min}$ and $70^{\circ} \mathrm{C}$ for $15 \mathrm{~min}$. Subsequently, first reaction mixture was added to the prepared RT mix containing 1.6 $\mu 1$ Nuclease-Free Water, $4.0 \mu \mathrm{l} \mathrm{GoScript}^{\mathrm{TM}}$ 5X Reaction Buffer, $2 \mu \mathrm{l} \mathrm{MgCl}_{2}$ (25 mM), $0.4 \mu \mathrm{l}$ recombinant RNasin ${ }^{\circledR}$ Ribonuclease Inhibitor, and $1 \mu$ l GoScript ${ }^{\mathrm{TM}}$ Reverse Transcriptase. The whole reverse transcription was performed in a Gene Amp PCR System 9700 at $25^{\circ} \mathrm{C}$ for $5 \mathrm{~min}, 42^{\circ} \mathrm{C}$ for $60 \mathrm{~min}$, and $70^{\circ} \mathrm{C}$ for $15 \mathrm{~min}$. The control cDNA synthesis reaction, without reverse transcriptase enzyme, was performed to test that the extracted RNA was not contaminated with genomic DNA. cDNAs were stored at $-20^{\circ} \mathrm{C}$ until later use.

$q P C R$. The qPCR reactions were performed according to the manufacturer's protocol and our previous study (15) in the Gene Amp PCR System 9700. Briefly, qPCR amplification was performed with $2 \mathrm{X}$ GoTaq ${ }^{\circledR}$ qPCR Master mix (Promega Corporation) in a final volume of $20 \mu \mathrm{l}$. The reaction mixture consisted of $10 \mu \mathrm{l} 2 \mathrm{X}$ GoTaq $^{\circledR}$ qPCR Master mix, $0.3 \mu 110 \mu \mathrm{M}$ Universal Adaptor PCR Forward Primer, $0.3 \mu 110 \mu \mathrm{M}$ PCR Reverse Primer, $2 \mu \mathrm{l}$ cDNA template, and $7.4 \mu \mathrm{l}$ sterile distilled water. The qPCR reaction was initiated with 2 min incubation at $95^{\circ} \mathrm{C}$, followed by 40 cycles at $95^{\circ} \mathrm{C}$ for $15 \mathrm{sec}$ and $60 \mathrm{sec}$ at $60^{\circ} \mathrm{C}$. Subsequently, a melting curve was performed at the end of the PCR run over a range of $55-99^{\circ} \mathrm{C}$, increasing the temperature stepwise by $0.5^{\circ} \mathrm{C}$ every $2 \mathrm{sec}$. The sample 


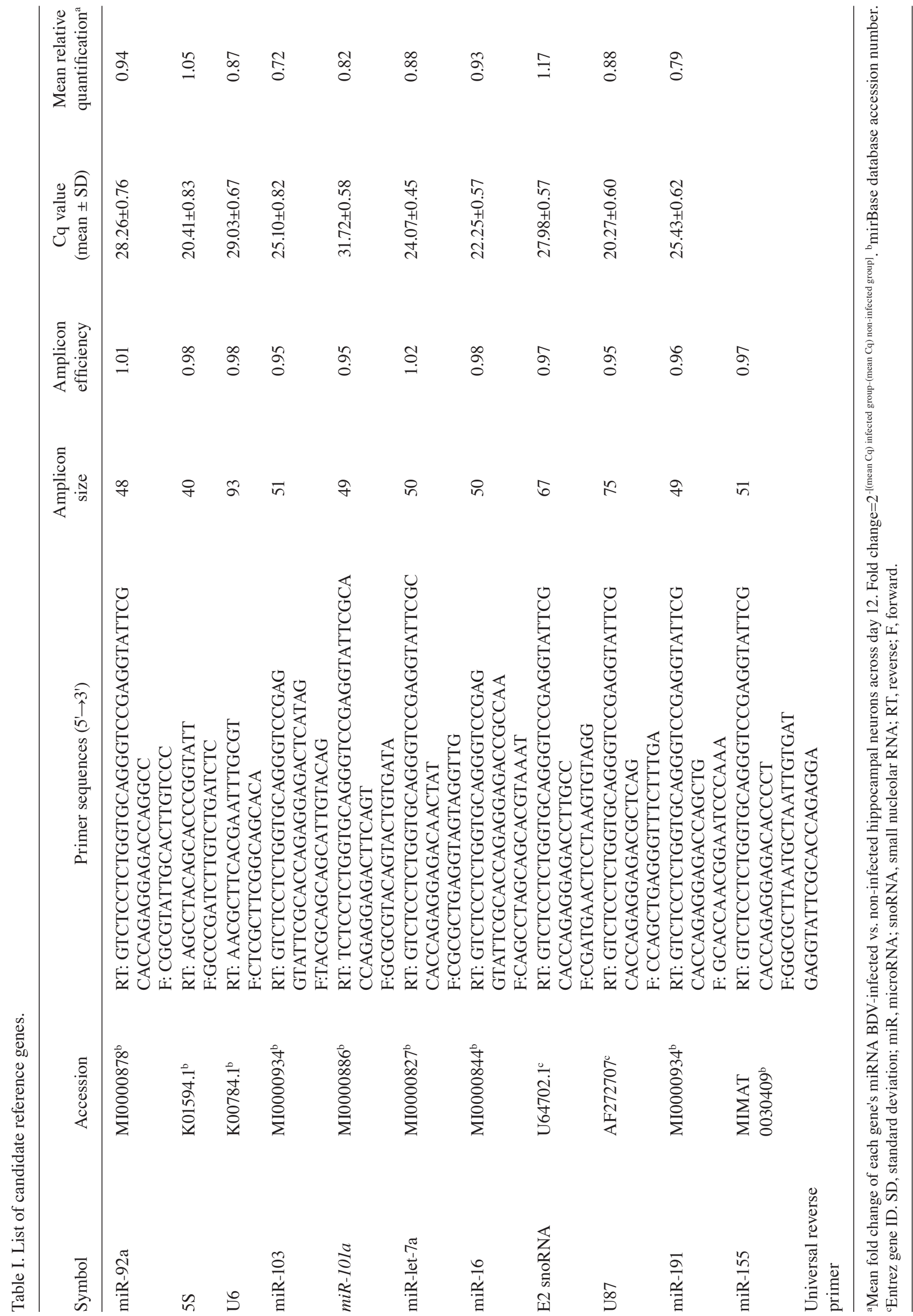



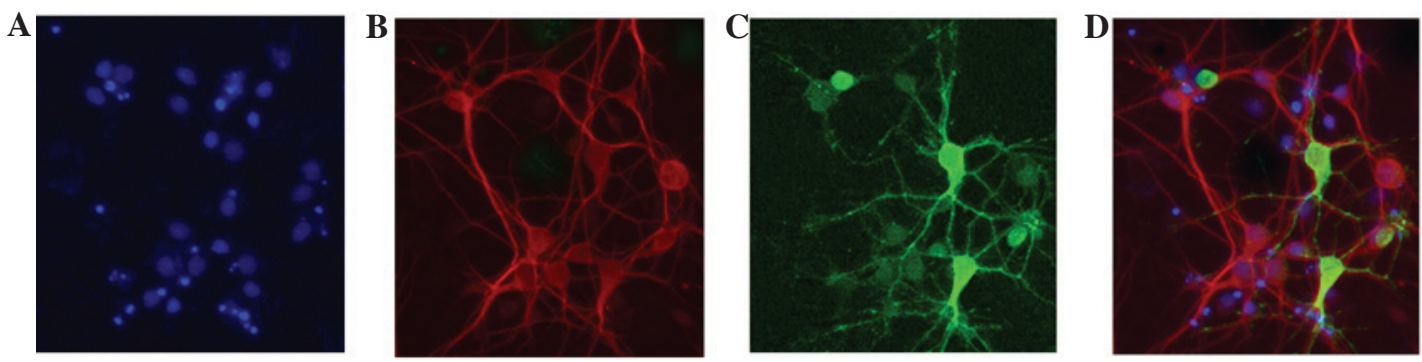

Figure 1. Immunofluorescent analysis of BDV-infected neurons on day 10. (A) Nuclei stained with DAPI (blue), (B) neurons marked with chicken polyclonal microtubule associated protein 2 followed by a Alexa Fluor 594-conjugated secondary antibody (red), (C) BDV P40 detected with a primary monoclonal antibody followed by a green fluorescein isothiocyanate-labeled secondary antibody and (D) merged image.

Table II. Expression stability of reference genes as calculated by geNorm, NormFinder, BestKeeper, and $\Delta-\mathrm{Ct}(\mathrm{c}+\mathrm{V}, \mathrm{n}=32)$.

\begin{tabular}{llllll}
\hline geNorm rank & Gene & M-value & NormFinder rank & Gene & Variability \\
\hline 1 & miR-101a & 0.3160 & 1 & U87 & 0.0799 \\
2 & U87 & 0.3160 & 2 & miR-92ap & 0.1186 \\
3 & miR-16 & 0.3662 & 3 & miR-101a & 0.1248 \\
4 & miR-let-7a & 0.3804 & 4 & miR-16 & 0.1265 \\
5 & miR-191 & 0.4167 & 5 & U6 & 0.1315 \\
6 & miR-103 & 0.4431 & 6 & miR-let-7a & 0.1384 \\
7 & U6 & 0.4773 & 7 & miR-191 & 0.1874 \\
8 & miR-92a & 0.5048 & 8 & 5 S & 0.2346 \\
9 & E2 snoRNA & 0.5566 & 9 & miR-103 & 0.3088 \\
10 & 5S & 0.6061 & 10 & E2 snoRNA & 0.3482 \\
\hline BestKeeper rank & Gene & Coeff. of corr.[r] & $\Delta-$ Ct rank & Gene & MeanStdDev \\
\hline 1 & U87 & 0.891 & 1 & miR-101a & 0.5154 \\
2 & miR-101a & 0.878 & 2 & U87 & 0.5167 \\
3 & miR-92a & 0.859 & 3 & miR-16 & 0.5538 \\
4 & miR-103 & 0.85 & 4 & miR-let-7a & 0.5704 \\
5 & miR-16 & 0.813 & 5 & miR-191 & 0.5769 \\
6 & U6 & & 6 & U6 & 0.6058 \\
7 & miR-191 & 0.797 & 7 & miR-92a & 0.6259 \\
8 & miR-let-7a & 0.724 & 8 & miR-103 & 0.6418 \\
9 & 5S & 0.633 & 10 & E2 snoRNA & 0.7528 \\
10 & E2 snoRNA & 0.427 & $5 \mathrm{~S}$ & 0.8176 \\
\hline
\end{tabular}

$\mathrm{C}+\mathrm{V}$ represents the control group and infected group, $n$ represents the number of independent samples. miR, microRNA; snoRNA, small nucleolar RNA.

maximization method criterion was used to establish the run layout. A dilution series was created with random cDNA from the sample group to construct standard curves for each primer pair. Gene-specific amplification was confirmed by a single peak in the melting-curve analysis, and a single band on a $2 \%$ agarose gel stained with ethidium bromide. All samples (each group, $n=16$; total, $n=32$ ) were diluted 20 times and the reaction was performed, as described above, in duplicate.

Data analysis. All statistical analyses were performed with SPSS 21.0 (IBM SPSS, Armonk, NY, USA) and the figures were produced with Graphpad Prism 5.0 (GraphPad
Software Inc., La Jolla, CA, USA). A Student's t-test and the Wilcoxon-Mann-Whitney test was used to determine statistically significant differences between the two groups. $\mathrm{P}<0.05$ was considered to indicate a statistically significant difference. Data were presented as the mean \pm standard error of the mean. The stability of miRNA expression for each reference gene was statistically analyzed with the four software packages, geNorm (https://genorm.cmgg.be/), NormFinder (http://moma. dk/normfinder-software), BestKeeper (http://www.gene-quantification.de/bestkeeper.html), and the comparative $\Delta-\mathrm{Ct}$ method (21). Cq values were transformed into relative expression quantity (RQ) values according to the $\Delta-C$ t formula 

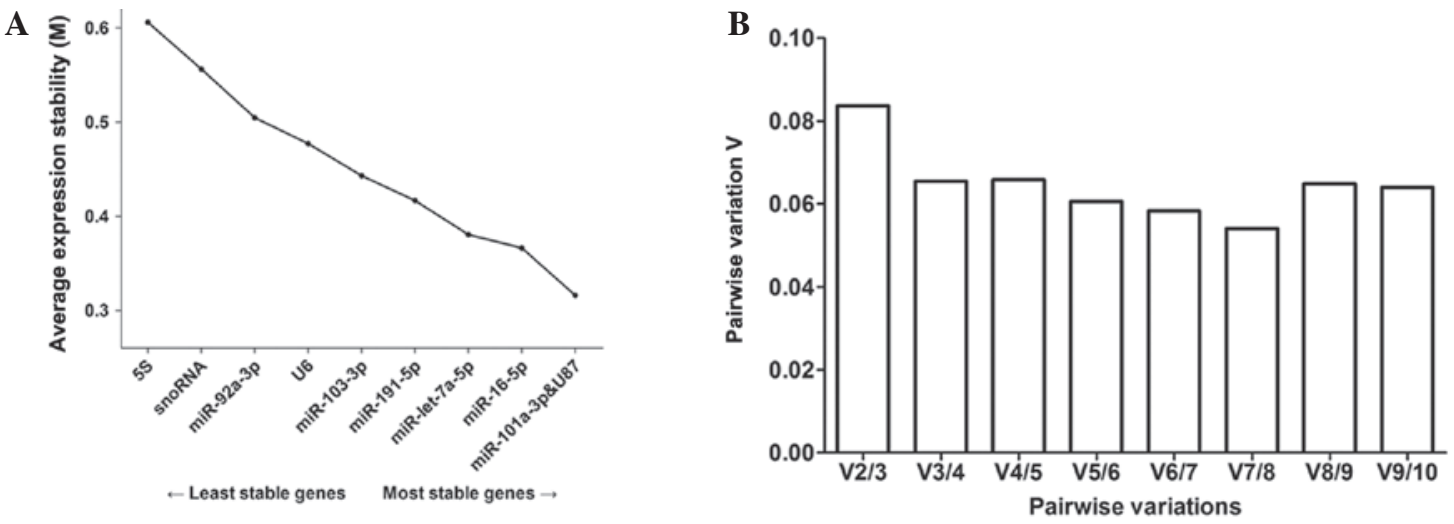

Figure 2. Mean expression stability measures calculated by geNorm. (A) Ranking of candidate reference genes according to mean expression stability (M-value). A lower M-value of the mean expression stability indicates more stable expression. (B) Determination of the optimal number of reference genes for normalization on the basis of a pair-wise variation $(\mathrm{Vn} / \mathrm{n}+1)$ analysis. The software calculates the normalization factor from at least two genes. miR, microRNA.

Table III. Consensus ranking of ten candidate reference genes.

\begin{tabular}{|c|c|c|c|c|c|}
\hline Ranking & geNorm & NormFinder & BestKeeper & $\Delta-\mathrm{Ct}$ & Consensus ranking \\
\hline 1 & miR-101a and U87 & U87 & U87 & miR-101a & miR-101a \\
\hline 2 & - & miR-92a & miR-101a- & U87 & U87 \\
\hline 3 & $\operatorname{miR}-16$ & miR-101a & miR-92a & miR-16- & miR-16 \\
\hline 4 & miR-let-7a & miR-16 & miR-103 & miR-let-7a & miR-92a \\
\hline 5 & miR-191 & U6 & miR-16 & miR-191 & miR-let-7a- \\
\hline 6 & $\operatorname{miR}-103$ & miR-let-7a & U6 & U6 & U6 \\
\hline 7 & U6 & miR-191 & miR-191 & miR-92a & miR-191 \\
\hline 8 & miR-92a & $5 \mathrm{~S}$ & miR-let-7a & miR-103 & miR-103 \\
\hline 9 & E2 snoRNA & miR-103 & $5 \mathrm{~S}$ & E2 snoRNA & $5 S$ \\
\hline 10 & $5 \mathrm{~S}$ & E2 snoRNA & E2 snoRNA & $5 \mathrm{~S}$ & E2 snoRNA \\
\hline
\end{tabular}

miR, microRNA; snoRNA, small nucleolar RNA.

Table IV. Relative gene expression ratios of miR-155.

\begin{tabular}{lcc}
\hline Reference genes & $\begin{array}{c}\text { miR-155 } \\
(\text { P-value, } n=16)\end{array}$ & $\begin{array}{c}2^{-\Delta \Delta C t} \text { value } \\
(\text { mean })\end{array}$ \\
\hline miR-101a & 0.000 & 0.695 \\
U87 & 0.000 & 0.702 \\
E2 snoRNA & 0.141 & 1.134 \\
\hline
\end{tabular}

miR, microRNA; snoRNA, small nucleolar RNA.

$\left.\left[R Q=E^{-\Delta C q}=E^{(\text {mean Cq-sample Cq) }}\right)\right]$ as required for inputs for the geNorm and NormFinder methods only. Subsequently, the stability measurements were combined to establish a consensus rank of the housekeeping genes using the RankAggreg package of the R project.

\section{Results}

Immunofluorescence assay. As our previous study (15) demonstrated that BDV $\mathrm{P} 40^{+}$neurons were $>80 \%$ at day 9 and almost $100 \%$ by day 12 , the cells were infected with a low MOI of 0.02 focus forming units and the current immunofluorescence assay was applied on day 10 . The purity of neurons and efficiency of infection was determined via observation of randomly selected cells across three independent experiments. On day 10, the results demonstrated that the purity of neurons was $>85 \%$ (Fig. $1 \mathrm{~A}$ and B). The infection efficiency of BDV $\mathrm{P} 40^{+}$neurons was $\sim 100 \%$ (Fig. $1 \mathrm{C}$ and D).

Evaluation of methods for measuring miRNAs in hippocampal neurons. To evaluate the expression level of these candidate reference genes in rat hippocampal neurons between the BDV-infected group and the control group at 12 days post-infection. RT-qPCR was used to detect and the gene products indicated single bands for all primer sets during agarose gel electrophoresis (data not shown). Melting curve analysis consistently demonstrated a single homogenous melting peak for each primer set and no amplicons were detected for the no template control. The amplification efficiencies for all candidate reference genes were indicated by the standard curve and are presented in Table I. These results indicated that the method of measurement was viable. 

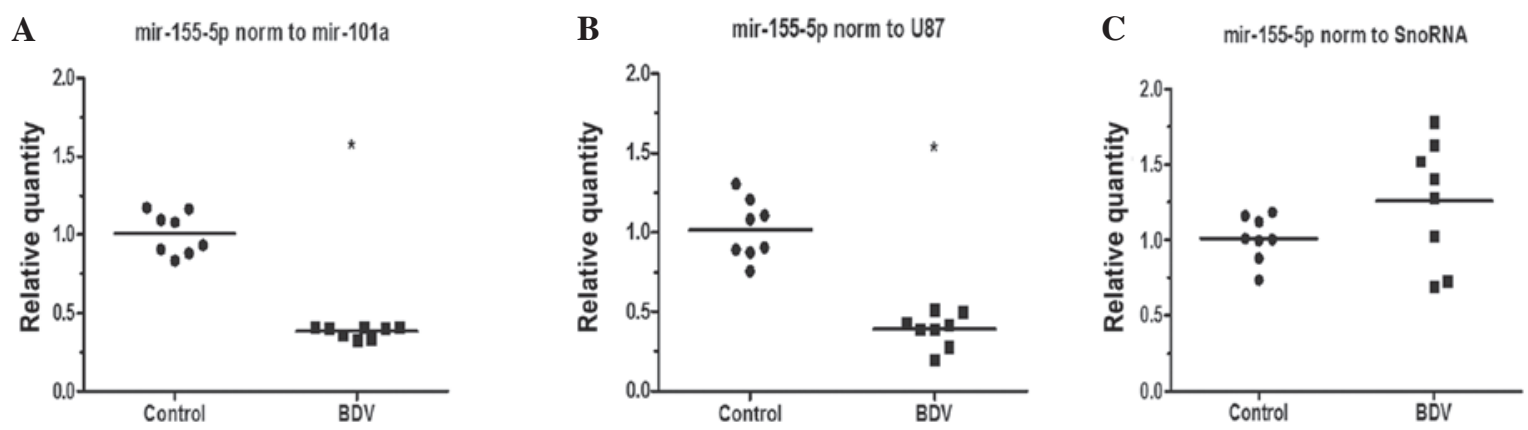

Figure 3. Relative gene expression ratios of miR-155. Assessment the validity of the selected reference genes using miR-155. Relative genes of mir-155 were normalized to (A) mir-101a, (B) U87 and (C) E2 snoRNA in non-infected and BDV-infected neurons. Scatter plots indicate the mean and standard error of the mean. BDV indicate BDV-infected neurons groups. "P<0.05 vs. the control. miR, microRNA; snoRNA, small nucleolar RNA; BDV, Borna disease virus.

Candidate reference gene ranking by expression stability. There were four aforementioned algorithms used to assess the stability of the candidate housekeeping gene miRNAs expression in infected cells and the control. GeNorm (22) provides a ranking of the candidate reference genes based on the their expression stability measure (M) in order to identify the most stable reference gene on day 12. The lowest M-value corresponds to the most stable reference gene, while the highest corresponds to the least stable one. In previous studies, an M-value $<0.5$ was set as a cut-off to assess gene stability in homogenous samples (23). GeNorm identified miR-101a and U87 (M-value, 0.3160; Table II) as the most stable pair-wise combination of reference genes for the experimental groups on day 12. Furthermore, geNorm calculated the pairwise variation $(\mathrm{Vn} / \mathrm{Vn}+1)$ that selects the optimum number of reference genes (Fig. 2). As reported by Vandesompele et al (24), the ideal pair-wise variation value is $<0.15$. As presented in Fig. 2 , the calculated V2/3 was $<0.15$. Thus, only the two most stable genes for a reliable normalization were taken into consideration. Subsequently, the most stable reference genes were identified by NormFinder (25), which enables the identification of the single most reliable gene and provides a ranking order. NormFinder identified U87 as the optimal reference gene on day 12 (Table II).

A total of 10 common used candidate reference genes were measured and $\Delta-\mathrm{Ct}(\mathrm{Cq}$, quantification cycle) analysis (26) demonstrated the ranking of the stability of reference genes by comparing the $\mathrm{Cq}$ value differences among all samples (Table II). The optimal reference genes were miR-101a and U87 with mean standard deviations of 0.5154 and 0.5167 , respectively, on day 12. BestKeeper (27) analyzes the correlation between the genes with the BestKeeper index based on a Pearson correlation (r) (20) using repeated pair-wise correlation analysis of candidate gene $\mathrm{Cq}$ values to determine optimal reference genes and recommended U87 (0.891) and miR-101a (0.898) as the two most stable genes.

Consensus ranking was performed by the RankAggreg package. The consensus ranking was compared with the rankings produced by geNorm, NormFinder, BestKeeper, and the comparative $\Delta$-Ct method (Table III). Thus, the RankAggreg output determined the most (miR-101a and U87) and the least (snoRNA) suitable reference genes.

Assessment of validity. To further investigate the use of miR-101a in miRNA relative quantification, miR-155 was selected as a standard by which to evaluate the use of miR-101a as a reference gene, as miR-155 has a core immune regulatory role in cells with BDV persistent infection, and miR-155 was observed to be downregulated in persistently infected BDV human oligodendroglia (OL/BDV) cells as the BDV P protein directly inhibited miR-155 expression (28). miRNA-155 regulates cell processes, including cell survival, growth, and chemosensitivity (29) and dendritic development and apoptosis (30). The relative expression levels of the target gene were evaluated using the most stable reference gene (miR-101a and U87) vs. the least reference stable gene (snoRNA). When using miR-101a and U87 as a reference gene, the relative expression of miR-155 was demonstrated to be significantly downregulated (Table IV and Fig. 3A and B; $\mathrm{P}<0.05)$, which is similar to a previous study (28). When snoRNA was selected as the reference gene, the relative expression levels of miR-155 was demonstrated to be upregulated (Table IV; Fig. 3C) and no significant difference was indicated between the BDV-infected and non-infected groups.

\section{Discussion}

miRNAs have been demonstrated to be important as regulators of gene expression, promoting increased understanding of gene regulation in normal development and in disease. miRNA expression profiles have been suggested be more accurate in disease classification than mRNA expression profiles (31). miRNAs are of research interest due to their role in the molecular changes underlying different disease models. miRNAs reduce expression of proteins encoded by their target RNAs, by binding to the 3'-untranslated region of target mRNAs. Thus, it is important to expand BDV research in this area, particularly in BDV-infected hippocampal neurons as it is an important part of brain.

Recently, human BDV infections have been reported in China and research into human infections BDV has been conducted (32-35). BDV is known to result in behavioral disturbances in mammals, however, its effects on humans is subject to debate (5). Furthermore, the present study is the first systematic comparison of different normalization approaches using RT-qPCR data in BDV-infected primary rat hippocampal neurons. To validate the appropriate reference genes, the expressions of 10 commonly used candidate 
reference genes were detected and analyzed were detected and analyzed. It was based on a three-step approach with RT-qPCR validation, selection of housekeeping miRNAs using computer software, and a proof of principle study with various miRNA expression. During the RT-qPCR validation stage, no candidate miRNAs were excluded from subsequent analysis. Thus, all 10 candidate reference genes were included in the geNorm, NormFinder, BestKeeper, and comparative $\Delta$-Ct analysis.

geNorm, NormFinder and BestKeeper analysis did not recommend the same reference genes for normalization (Table III). This may be due to the different statistical outputs of the software, namely M-values obtained from geNorm, variability measurements from NormFinder, coefficients of correlation from BestKeeper and mean standard deviations from the $\Delta-\mathrm{Ct}$ method. The RankAggreg software package was used to analyze the four different sets of data and combine these four algorithms to establish a consensus ranking. Specifically, the brute force method (using the RankAggreg function) was used to enumerate all possible candidate lists and then the one with the minimum Spearman foot rule distance was selected (36). This method output results in the most stable and least stable reference genes on day 12 , however, there may not be consistency in the expression levels of certain reference genes over time under certain conditions $(22,37)$. Based on the different normalization strategies, miR-101a was determined to be the most optimal and stable reference gene. Furthermore, mir-101a was also demonstrated to be ideal for normalization of serum microRNA in gastric cancer patients (38).

There are a number of limitations to the present study. Firstly, only 10 commonly used candidate housekeeping genes were analyzed, whereas there may exist more superior combinations of housekeeping genes for BDV research in BDV infection cell lines. Secondly, only one cell type was used in vitro (rat cortical neurons) was investigated for the suitability of reference genes for RT-qPCR, and RNA extracted from in vivo BDV-infected rat brains was not analyzed. However, parameters in neuron cultures have been standardized in the present study, and neuron cultures are the major cellular targets for BDV in nature, thus, the current study provides important information to advance BDV research.

In conclusion, housekeeping gene expression levels were comparatively evaluated for the normalization of miRNAs in BDV research by RT-qPCR. The combined use of miR-101 and U87 was assessed as the optimal normalization method for miRNA expression data from hippocampal neurons infected BDV. Assessment of the validity of the selected reference genes confirms the suitability of applying a combination of the most stable reference genes. The present study provides a method by which more reliable and accurate gene expression measurements can be obtained, and it has identified a reliable two gene (miR-101 and U87) normalizer for use in this context.

\section{Acknowledgements}

The present study was supported by the National Natural Science Foundation of China (grant no. 31300137), the Medical
Scientific Research Project of Chongqing Health Bureau (grant no. 20142022), the National Key Scientific Program of China (grant nos. 2009CB918300 and 2012CB910602). The authors would like to thank the scientific editors at Impactys (www.impactys.com) for editing and proofreading this manuscript.

\section{References}

1. de la Torre JC: Molecular biology of borna disease virus: Prototype of a new group of animal viruses. J Virol 68: 7669-7675, 1994

2. Ludwig H, Bode L and Gosztonyi G: Borna disease: A persistent virus infection of the central nervous system. Prog Med Virol 35: 107-151, 1988.

3. Kinnunen PM, Palva A, Vaheri A and Vapalahti O: Epidemiology and host spectrum of Borna disease virus infections. J Gen Virol 94: 247-262, 2013.

4. Zhang L, Xu MM, Zeng L, Liu S, Liu X, Wang X, Li D, Huang RZ, Zhao LB, Zhan QL, et al: Evidence for Borna disease virus infection in neuropsychiatric patients in three western China provinces. Eur J Clin Microbiol Infect Dis 33: 621-627, 2014.

5. Liu X, Bode L, Zhang L, Wang X, Liu S, Zhang L, Huang R, Wang M, Yang L, Chen S, et al: Health care professionals at risk of infection with Borna disease virus-evidence from a large hospital in China (Chongqing). Virol J 12: 39, 2015.

6. Gonzalez-Dunia D, Sauder C and de la Torre JC: Borna disease virus and the brain. Brain Res Bull 44: 647-664, 1997.

7. Pletnikov MV, Rubin SA, Moran TH and Carbone KM: Exploring the cerebellum with a new tool: Neonatal Borna disease virus (BDV) infection of the rat's brain. Cerebellum 2: 62-70, 2003.

8. Gosztonyi G: Natural and experimental Borna disease virus infections-neuropathology and pathogenetic considerations. APMIS Suppl 53-57, 2008.

9. Mestdagh P, Feys T, Bernard N, Guenther S, Chen C, Speleman F and Vandesompele J: High-throughput stem-loop RT-qPCR miRNA expression profiling using minute amounts of input RNA. Nucleic Acids Res 36: e143, 2008.

10. Beaudoin GM III, Lee SH, Singh D, Yuan Y, Ng YG, Reichardt LF and Arikkath J: Culturing pyramidal neurons from the early postnatal mouse hippocampus and cortex. Nat Protoc 7: 1741-1754, 2012

11. Bajramovic JJ, Münter S, Syan S, Nehrbass U, Brahic M and Gonzalez-Dunia D: Borna disease virus glycoprotein is required for viral dissemination in neuons. J Virol 77: 12222-12231, 2003.

12. Bode L, Dürrwald R, Rantam FA, Ferszt R and Ludwig H: First isolates of infectious human Borna disease virus from patients with mood disorders. Mol Psychiatry 1: 200-212, 1996.

13. Suberbielle E, Stella A, Pont F, Monnet C, Mouton E, Lamouroux L, Monsarrat B and Gonzalez-Dunia D: Proteomic analysis reveals selective impediment of neuronal remodeling upon Borna disease virus infection. J Virol 82: 12265-12279, 2008.

14. Huang R, Gao H, Zhang L, Jia J, Liu X, Zheng P, Ma L, Li W, Deng J, Wang X, et al: Borna disease virus infection perturbs energy metabolites and amino acids in cultured human oligodendroglia cells. PLoS One 7: e44665, 2012.

15. Zhang L, Liu S, Zhang L, You H, Huang R, Sun L, He P, Chen S, Zhang $\mathrm{H}$ and Xie P: Real-time qPCR identifies suitable reference genes for Borna disease virus-infected rat cortical neurons. Int J Mol Sci 15: 21825-21839, 2014.

16. Lewin GR and Barde YA: Physiology of the neurotrophins. Annu Rev Neurosci 19: 289-317, 1996.

17. Davoren PA, McNeill RE, Lowery AJ, Kerin MJ and Miller N: Identification of suitable endogenous control genes for microRNA gene expression analysis in human breast cancer. BMC Mol Biol 9: 76, 2008.

18. Lardizabal MN, Nocito AL, Daniele SM, Ornella LA, Palatnik JF and Veggi LM: Reference genes for real-time PCR quantification of microRNAs and messenger RNAs in rat models of hepatotoxicity. PLoS One 7: e36323, 2012.

19. Ratert N, Meyer HA, Jung M, Mollenkopf HJ, Wagner I, Miller K, Kilic E, Erbersdobler A, Weikert S and Jung K: Reference miRNAs for miRNAome analysis of urothelial carcinomas. PLoS One 7: e39309, 2012. 
20. Liu X, Zhang L, Cheng K, Wang X, Ren G and Xie P. Identification of suitable plasma-based reference genes for miRNAome analysis of major depressive disorder. J Affect Disord 163: 133-139, 2014.

21. Wang Y, Tang N, Hui T, Wang S, Zeng X, Li H and Ma J: Identification of endogenous reference genes for RT-qPCR analysis of plasma microRNAs levels in rats with acetaminophen-induced hepatotoxicity. J Appl Toxicol 33: 1330-1336, 2013.

22. Langnaese K, John R, Schweizer H, Ebmeyer U and Keilhoff G: Selection of reference genes for quantitative real-time PCR in a rat asphyxial cardiac arrest model. BMC Mol Biol 9: 53, 2008

23. Kim I, Yang D, Tang X and Carroll JL: Reference gene validation for $\mathrm{qPCR}$ in rat carotid body during postnatal development. BMC Res Notes 4: 440, 2011.

24. Vandesompele J, De Preter K, Pattyn F, Poppe B, Van Roy N, De Paepe A and Speleman F: Accurate normalization of real-time quantitative RT-PCR data by geometric averaging of multiple internal control genes. Genome Biol 3: RESEARCH0034, 2002.

25. Zhao C, Zha Y, Wu X, Chen L, Shi J and Cui L: The quantification of ADAMTS4 and 8 expression and selection of reference genes for quantitative real-time PCR analysis in myocardial infarction. Biomed Pharmacother 65: 555-559, 2011.

26. Silver N, Best S, Jiang J and Thein SL: Selection of housekeeping genes for gene expression studies in human reticulocytes using real-time PCR. BMC Mol Biol 7: 33, 2006.

27. Pfaffl MW,Tichopad A,PrgometC and NeuviansTP:Determination of stable housekeeping genes, differentially regulated target genes and sample integrity: BestKeeper-Excel-based tool using pair-wise correlations. Biotechnol Lett 26: 509-515, 2004.

28. Zhai A, Qian J, Kao W, Li A, Li Y, He J, Zhang Q, Song W, Fu Y, $\mathrm{Wu} \mathrm{J}$, et al: Borna disease virus encoded phosphoprotein inhibits host innate immunity by regulating miR-155. Antiviral Res 98 : 66-75, 2013.

29. Kong W, He L, Coppola M, Guo J, Esposito NN, Coppola D and Cheng JQ: MicroRNA-155 regulates cell survival, growth, and chemosensitivity by targeting FOXO3a in breast cancer. J Biol Chem 285: 17869-17879, 2010.
30. Lu C, Huang X, Zhang X, Roensch K, Cao Q, Nakayama KI, Blazar BR, Zeng Y and Zhou X: miR-221 and miR-155 regulate human dendritic cell development, apoptosis, and IL-12 production through targeting of p27kip1, KPC1, and SOCS-1. Blood 117: 4293-4303, 2011.

31. Lu J, Getz G, Miska EA, Alvarez-Saavedra E, Lamb J, Peck D, Sweet-Cordero A, Ebert BL, Mak RH, Ferrando AA, et al: MicroRNA expression profiles classify human cancers. Nature 435: 834-838, 2005.

32. Liu X, Yang Y, Zhao M, Bode L, Zhang L, Pan J, Lv L, Zhan Y, Liu S, Zhang L, et al: Proteomics reveal energy metabolism and mitogen-activated protein kinase signal transduction perturbation in human Borna disease virus $\mathrm{Hu}-\mathrm{H} 1$-infected oligodendroglial cells. Neuroscience 268: 284-296, 2014.

33. Liu X, Zhao L, Yang Y, Bode L, Huang H, Liu C, Huang R, Zhang L, Wang X, Zhang L, et al: Human borna disease virus infection impacts host proteome and histone lysine acetylation in human oligodendroglia cells. Virology 464-465: 196-205, 2014.

34. Li D, Lei Y, Deng J, Zhou C, Zhang Y, Li W, Huang H, Cheng S, Zhang $\mathrm{H}$, Zhang L, et al: Human but not laboratory Borna disease virus inhibits proliferation and induces apoptosis in human oligodendrocytes in vitro. PLoS One 8: e66623, 2013.

35. Li Q, Wang Z, Zhu D, Xu M, Chen X, Peng D, Iwata Y and Xie P: Detection and analysis of Borna disease virus in Chinese patients with neurological disorders. Eur J Neurol 16: 399-403, 2009.

36. Pihur V, Datta S and Datta S: RankAggreg, an R package for weighted rank aggregation. BMC Bioinformatics 10: 62, 2009.

37. Lilly ST, Drummond RS, Pearson MN and MacDiarmid RM: Identification and validation of reference genes for normalization of transcripts from virus-infected Arabidopsis thaliana. Mol Plant Microbe Interact 24: 294-304, 2011.

38. Song J, Bai Z, Han W, Zhang J, Meng H, Bi J, Ma X, Han S and Zhang Z: Identification of suitable reference genes for qPCR analysis of serum microRNA in gastric cancer patients. Dig Dis Sci 57: 897-904, 2012. 